\title{
Excitations and finite-size properties of an integrable supersymmetric electronic model
}

\author{
A. L. Malvezzi \\ Departamento de Física, Faculdade de Ciências, UNESP - Universidade Estadual Paulista, CP 473, 17033-360 Bauru, SP, Brazil
}

M. J. Martins

Departamento de Física, Universidade Federal de São Carlos, CP 676, 13565-905 São Carlos, SP, Brazil (Received 13 October 2009; revised manuscript received 30 November 2009; published 27 January 2010)

\begin{abstract}
We investigate the bulk and finite-size properties of an integrable extension of the Hubbard model with a free parameter $\gamma$ related to the quantum deformation of the superalgebra $\operatorname{sl}(2 \mid 2)^{(2)}$. The Bethe ansatz solution is used to determine the ground state and the nature of the spin and charge excitations. As $\gamma \rightarrow 0$ the charge dispersion relation for low momenta crossover from linear to parabolic foreseeing a change from antiferromagnetic to ferromagnetic behavior. The study of the finite-size corrections to the spectrum reveals us that the underlying conformal theory has central charge $c=-1$ and a related effective central charge $c_{e f}=2$ as well as critical exponents depending on the parameter $\gamma$. We note that exact results at the isotropic point $\gamma=0$ can be established without recourse to the Bethe ansatz solution.
\end{abstract}

DOI: 10.1103/PhysRevB.81.035120

PACS number(s): 71.10.Fd, 75.10.Jm, 71.10.Pm, 64.60.an

\section{INTRODUCTION}

The study of electron correlation effects in onedimensional systems has by now attracted the attention of theorists for more than half-century. The physical behavior of one-dimensional correlated electron models are expected to be drastically different from that of free electrons. ${ }^{1}$ The corresponding elementary excitations have a collective character and nonperturbative techniques are essential to unveil the physical behavior. In this sense, electronic lattice systems solvable by the Bethe ansatz, such as the Hubbard model ${ }^{2}$ and quantum lattice gases, ${ }^{3}$ have provided relevant insights in the understanding of correlation effects in low dimensions. ${ }^{4}$

Of particular interest are integrable extensions of Hubbard model containing extra correlation mechanisms besides the standard charge-charge interactions among electrons. These models can be derived exploring solutions of the graded Yang-Baxter equation ${ }^{5}$ with two fermionic and two bosonic degrees of freedom. The respective Hamiltonian is built up from the logarithmic derivative of the Yang-Baxter solution based on a given Lie superalgebra. The Hamiltonian can then be made invariant by the underlying Lie superalgebra and in the scope of condensed matter such invariance is often referred as supersymmetries. ${ }^{6,7}$ This invariance does not contain the Lorentz group and therefore should not be confused with spacetime supersymmetry of field theories. We remark however that fermionic lattice models with underlying spacetime supersymmetry have also been proposed and investigated in the literature. ${ }^{8}$ Their Hamiltonians are derived from the anticommutator of supersymmetric generators providing for instance a number of exact results for the spectrum. ${ }^{9}$ For a review of the properties of this class of models and further references see Ref. 10.

To what concerns extended Hubbard models arising from supergroups, representative examples are the models based on the four dimensional representations of the $\operatorname{sl}(2 \mid 2)$ and $\operatorname{gl}(2 \mid 1)$ Lie superalgebras. ${ }^{6,7,11,12}$ We remark that further generalizations can be constructed by means of the quantum deformations of such algebras ${ }^{13-15}$ as well as on the central extension of $\operatorname{sl}(2 \mid 2) .{ }^{17}$ The purpose of this paper is to investigate the critical properties of an extended Hubbard model based on the quantum deformation of the twisted $\operatorname{sl}(2 \mid 2)^{(2)}$ algebra. ${ }^{14} \mathrm{We}$ recall here that this model appears to provide a lattice regularization of an interesting integrable $(1+1)$-dimensional quantum field of two coupled massive Dirac fermions. ${ }^{16}$ Though the respective Bethe ansatz solution is known ${ }^{15}$ it has not yet been explored to extract information about the physical properties of such lattice electronic model. Following ${ }^{15}$ the model Hamiltonian can be rewritten as

$$
\begin{aligned}
H= & \sum_{i=1}^{L} \sum_{\sigma= \pm}\left[c_{i, \sigma}^{\dagger} c_{i+1, \sigma}+\text { H.c. }\right]\left[1-X_{\sigma} n_{i,-\sigma}-\bar{X}_{\sigma} n_{i+1,-\sigma}\right] \\
& +U \sum_{i=1}^{L} n_{i,+} n_{i,-}+V \sum_{i=1}^{L}\left[n_{i,+} n_{i+1,-}+n_{i,-} n_{i+1,+}\right] \\
& +Y \sum_{i=1}^{L}\left[c_{i,+}^{\dagger} c_{i,-}^{\dagger} c_{i+1,-} c_{i+1,+}+\text { H.c. }\right] \\
& +J \sum_{i=1}^{L}\left[c_{i,+}^{\dagger} c_{i+1,-}^{\dagger} c_{i,-} c_{i+1,+}+\text { H.c. }\right]-\mu \sum_{i=1}^{L}\left[n_{i,+}+n_{i,-}\right]
\end{aligned}
$$

where $c_{i, \sigma}^{\dagger}$ and $c_{i, \sigma}$ are fermionic creation and annihilation operators with spin index $\sigma= \pm$ acting on a chain of length $L$. The operator $n_{i, \sigma}=c_{i, \sigma}^{\dagger} c_{i, \sigma}$ represents the number of electrons with spin $\sigma$ on the $i$ th site.

Apart from the standard kinetic hopping amplitude and the on-site Coulomb term $U$ we see that Hamiltonian (1) contains additional interaction terms. They are the bondcharge hopping amplitudes $X_{\sigma}$ and $\bar{X}_{\sigma}$, the Coulomb interaction $V$ among electrons at nearest-neighbor sites, the spinspin exchange term $J$, the pair-hopping amplitude $Y$ besides the chemical potential amplitude $\mu$. Integrability constraints the couplings of the model on the following one-parameter manifold: 


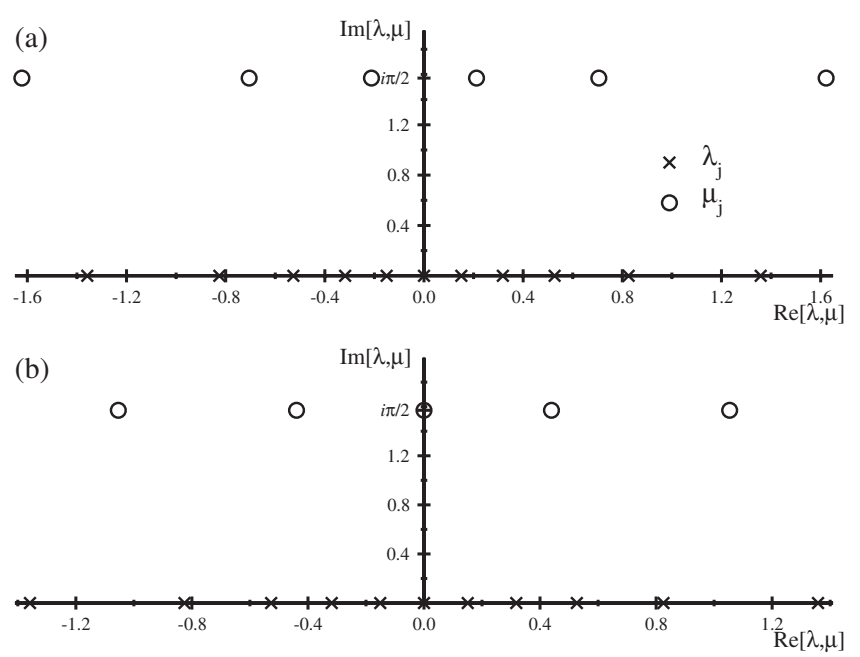

FIG. 1. The ground state roots $\lambda_{j}$ (crosses) and $\mu_{j}$ (circles) for $\gamma=\pi / 5$ and $L=12$ in sectors (a) $N_{+}=L / 2, N_{-}=L / 2-1$ and (b) $N_{+}=L / 2-1, N_{-}=L / 2$. We note that the roots $\lambda_{j}$ are the same for both sectors.

$$
\begin{gathered}
X_{\sigma}=1+\sigma \sin (\gamma), \quad \bar{X}_{\sigma}=1-\sigma \sin (\gamma), \\
\frac{U}{2}=V=J=Y=\cos (\gamma),
\end{gathered}
$$

where the anisotropy $\gamma$ is related to the $q$ deformation of $\operatorname{sl}(2 \mid 2)^{(2)}$ by $q=\exp [i \gamma]$.

The potential $\mu$ is in principle arbitrary since the model conserves the total number of electrons with spin $\sigma= \pm$. However, the invariance of Hamiltonian (1) by the superalgebra $U_{q}\left[\mathrm{sl}(2 \mid 2)^{(2)}\right]$ fixes a relation between $\mu$ and $\gamma$, namely, ${ }^{14,15}$

$$
\mu=2 \cos (\gamma) \text {. }
$$

Considering parametrizations (2) and (3) one can relate the spectra of Hamiltonian Eq. (1) at the points $\gamma$ and $\pi-\gamma$. In fact, by performing a combination of particle-hole $c_{i, \sigma}$ $\rightarrow c_{i, \sigma}^{\dagger}$ and the parity $c_{i, \sigma} \rightarrow(-1)^{i} c_{i, \sigma}$ transformations one is able to find the following relation:

$$
H(\gamma)=-H(\pi-\gamma) \text {. }
$$

Due to property (4) the analysis of the physical properties of Hamiltonian (1) subjected to the constraints $(2,3)$ can be restricted to the antiferromagnetic interval $0 \leq \gamma \leq \pi / 2$. In this work we shall argue that the low-energy behavior of this model in the regime $0<\gamma \leq \pi / 2$ is that of a conformally invariant theory with central charge $c=-1$ and underlying effective central charge $c_{e f}=2$. The point $\gamma=0$ is special since the model reduces to the supersymmetric isotropic $\operatorname{sl}(2 \mid 2)$ extended Hubbard model. ${ }^{6}$ In this case it was argued that though the excitations are gapless the dispersion relations have a nonrelativistic branch. ${ }^{18,19}$ In fact, we found that for the electronic model (1)-(3) the speed of sound of the underlying low-lying excitations is proportional to $\sin (\gamma)$ which vanishes in the $\gamma \rightarrow 0$ limit.

We have organized this paper as follows. In next section we shall explore the Bethe ansatz solution to determine the

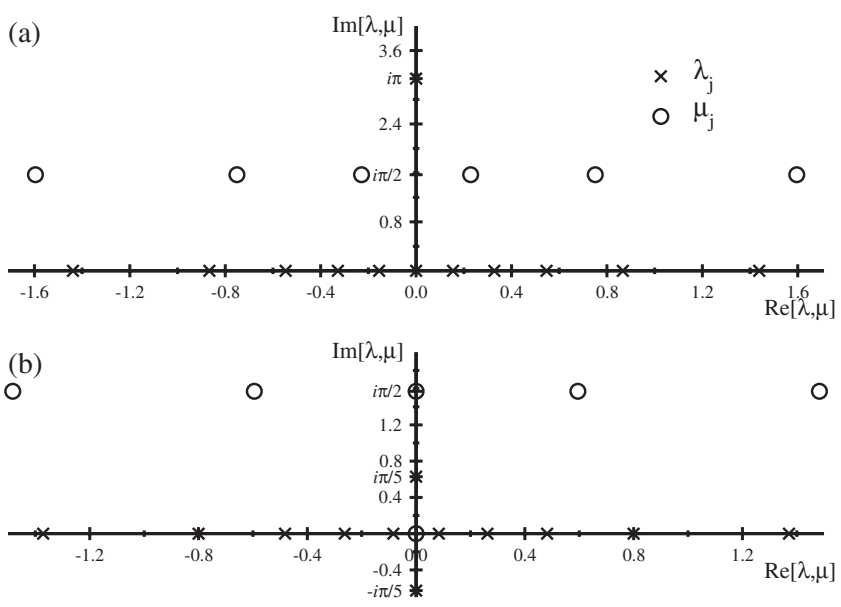

FIG. 2. The first excited state roots $\lambda_{j}$ (crosses) and $\mu_{j}$ (circles) for $\gamma=\pi / 5$ and $L=12$. Note that (b) has two roots $\lambda_{j}$ fixed at $\pm i \pi / 5$.

ground state and the nature of the excitations of the electronic model (1)-(3). A particular characteristic is that the dispersion relation of charge excitations combines both the behavior of massless and massive degrees of freedom. We note that as $\gamma \rightarrow 0$ the charge dispersion for low energies changes its form from linear to quadratic dependence on the momenta and a corresponding change on the ground state behavior. In Sec. III we study that finite-size properties of the spectrum of Hamiltonian (1)-(3) by both analytical and numerical approach. We argue that the critical properties are described by a critical line with central charge $c=-1$. Our conclusions are summarized in Sec. IV.

\section{THERMODYNAMIC LIMIT}

Here we will determine the ground state and the nature of the elementary excitations of the electronic model of Sec. I. These properties can be investigated by exploring the diagonalization of Hamiltonians (1)-(3) by the Bethe ansatz method. It was found that the corresponding spectrum is parameterized by the following nested Bethe equations: ${ }^{15}$

$$
\begin{gathered}
{\left[\frac{\sinh \left(\lambda_{j} / 2-i \gamma / 2\right)}{\sinh \left(\lambda_{j} / 2+i \gamma / 2\right)}\right]^{L}=\prod_{k=1}^{N_{+}} \frac{\sinh \left(\lambda_{j}-\mu_{k}-i \gamma\right)}{\sinh \left(\lambda_{j}-\mu_{k}+i \gamma\right)},} \\
j=1, \ldots, N_{+}+N_{-}
\end{gathered}
$$

and

$$
\begin{gathered}
\prod_{k=1}^{N_{+}+N_{-}} \frac{\sinh \left(\mu_{j}-\lambda_{k}-i \gamma\right)}{\sinh \left(\mu_{j}-\lambda_{k}+i \gamma\right)}=-\prod_{k=1}^{N_{+}} \frac{\sinh \left(\mu_{j}-\mu_{k}-2 i \gamma\right)}{\sinh \left(\mu_{j}-\mu_{k}+2 i \gamma\right)}, \\
j=1, \ldots, N_{+},
\end{gathered}
$$

where the integers $N_{\sigma}$ denote the total number of electrons with spin $\sigma= \pm$.

The eigenvalues $E(L, \gamma)$ of Hamiltonian (1)-(3) are given in terms of the variables $\lambda_{j}$ by 


$$
E(L, \gamma)=\sum_{j=1}^{N_{+}+N_{-}} \frac{2 \sin ^{2}(\gamma)}{\cos (\gamma)-\cosh \left(\lambda_{j}\right)}
$$

To make further progress it is important to identify the distribution of roots $\left\{\lambda_{j}, \mu_{k}\right\}$ on the complex plane which reproduce the low-lying energies of Hamiltonian (1)-(3). This task is performed by first determining the particle number sectors of the low-lying eigenvalues. This is done by means of brute force diagonalization of the Hamiltonian for small chains $L \leq 12$ and a few values of the parameter $\gamma$. We then compare these eigenvalues with the results coming from the numerical analysis of the solutions of the Bethe ansatz equations (5)-(7). By performing this analysis we find that the ground state in the regime $0<\gamma \leq \pi / 2$ for $L$ even sits, for periodic boundary conditions, in sectors $N_{+}$ $=L / 2 \pm 1, N_{-}=L / 2$ or $N_{+}=L / 2, N_{-}=L / 2 \pm 1$ and therefore it is fourfold degenerated. Due to the particle-hole symmetry it is sufficient to determine the respective pattern of the Bethe roots $\left\{\lambda_{j}, \mu_{k}\right\}$ for the sector with the minimum possible number of roots. In Fig. 1 we exhibit the ground state Bethe roots for $L=12$ in sectors $N_{+}=L / 2, N_{-}=L / 2-1$ and $N_{+}$ $=L / 2-1, N_{-}=L / 2$. We clearly see that the roots $\lambda_{j}$ are real while $\mu_{k}$ have a fixed imaginary part at $i \pi / 2$. The first excited state is double degenerated and lies in sector $N_{+}=N_{-}$ $=L / 2$. In Fig. 2 we show the corresponding Bethe roots $\left\{\lambda_{j}, \mu_{k}\right\}$ for $L=12$. By performing this analysis for the lowenergy excitations we find that they can be described mostly in terms of real variables when the second Bethe roots $\mu_{k}$ is shifted by the complex number $i \pi / 2$. Considering this discussion we find convenient to introduce the following variables:

$$
\lambda_{j}=\lambda_{j}^{(1)}, \quad \mu_{j}=\lambda_{j}^{(2)}+i \frac{\pi}{2},
$$

where $\lambda_{j}^{(a)} \in \mathfrak{R}$ for $a=1,2$.

Now by substituting Eq. (8) in the Bethe ansatz equations (5) and (6) and afterward by taking their logarithms we find that the resulting relations for $\lambda_{j}^{(a)}$ are

$$
\begin{gathered}
L \Phi\left(\frac{\lambda_{j}^{(1)}}{2}, \frac{\gamma}{2}\right)=2 \pi Q_{j}^{(1)}-\sum_{k=1}^{N_{+}} \Phi\left(\lambda_{j}^{(1)}-\lambda_{k}^{(2)}, \frac{\pi}{2}-\gamma\right), \\
j=1, \ldots, N_{+}+N_{-}
\end{gathered}
$$

and

$$
\begin{gathered}
-\sum_{\substack{k=1 \\
k \neq j}}^{N_{+}} \Phi\left(\lambda_{j}^{(2)}-\lambda_{k}^{(2)}, 2 \gamma\right)+2 \pi Q_{j}^{(2)} \\
=\sum_{k=1}^{N_{+}+N_{-}} \Phi\left(\lambda_{j}^{(2)}-\lambda_{k}^{(1)}, \frac{\pi}{2}-\gamma\right), \\
j=1, \ldots, N_{+}
\end{gathered}
$$

where function $\Phi(\lambda, \gamma)=2 \arctan [\cot (\gamma) \tanh (\lambda)]$.

The numbers $Q_{j}^{(a)}$ define the many possible logarithm branches and in general are integers or half-integers. Considering our previous numerical analysis we find that the lowlying spectrum is well described by the following sequence of $Q_{j}^{(a)}$ numbers:

$$
\begin{gathered}
Q_{j}^{(1)}=-\frac{1}{2}\left[L-n_{+}-n_{-}-1\right]+j-1, \quad j=1, \ldots, L-n_{+}-n_{-}, \\
Q_{j}^{(2)}=-\frac{1}{2}\left[\frac{L}{2}-n_{+}-1\right]+j-1, \quad j=1, \ldots, \frac{L}{2}-n_{+},
\end{gathered}
$$

where $n_{ \pm}$are integers labeling the sector with $N_{ \pm}=L / 2$ $-n_{ \pm}$particles with spin $\sigma= \pm$.

For large $L$ the number of roots tend toward a continuous distribution on the real axis whose density can be defined in terms of the counting function $Z\left(\lambda_{j}^{(a)}\right)=Q_{j}^{(a)} / L$ by the expression

$$
\rho^{(a)}\left(\lambda^{(a)}\right)=\frac{d Z\left(\lambda_{j}^{(a)}\right)}{d \lambda_{j}^{(a)}}, \quad a=1,2 .
$$

In the thermodynamic $L \rightarrow \infty$ limit the Bethe equations (9) and (10) turn into coupled linear integral relations for the densities $\rho^{(a)}\left(\lambda^{(a)}\right)$ which can be solved by the Fourier transform method. The final results for the densities are

$$
\begin{gathered}
\rho^{(1)}\left(\lambda^{(1)}\right)=\frac{2}{\pi\left[\cosh \left(2 \lambda^{(1)}\right)-\cos (2 \gamma)\right]}, \\
\rho^{(2)}\left(\lambda^{(2)}\right)=\frac{1}{2 \pi \cosh \left(\lambda^{(2)}\right)} .
\end{gathered}
$$

Now from the expressions for the density $\rho^{(1)}\left(\lambda^{(1)}\right)$ and Eq. (7) we can compute the ground state energy per site $e_{\infty}(\gamma)=\lim _{L \rightarrow \infty} E_{0}(L, \gamma) / L$. By writing the infinite volume limit of Eq. (7) in terms of its Fourier transform we find

$$
e_{\infty}(\gamma)=-4 \sin (\gamma) \int_{0}^{\infty} d \omega \frac{\cosh [\omega(\pi / 2-\gamma)] \sinh [\omega(\pi-\gamma)]}{\cosh [\omega \pi / 2] \sinh [\omega \pi]} \quad \text { for } 0<\gamma \leq \frac{\pi}{2}
$$


Let us consider the behavior of the low-lying excited states about the ground state. As usual these states are obtained from the Bethe equations (9) and (10) by making alternative choices of numbers $Q_{j}^{(a)}$ over the ground state configuration. This procedure is nowadays familiar to models solved by Bethe ansatz and for technical details see, for instance. ${ }^{3,20}$ It turns out that the expressions for the energy $\varepsilon^{(a)}\left(\lambda^{(a)}\right)$ and the momenta $p^{(a)}\left(\lambda^{(a)}\right)$, measured from the ground state, of a hole excitation on the $a$ th branch is given by

$$
\varepsilon^{(a)}\left(\lambda^{(a)}\right)=2 \pi \rho^{(a)}\left(\lambda^{(a)}\right), \quad p^{(a)}\left(\lambda^{(a)}\right)=\int_{\lambda^{(a)}}^{\infty} \varepsilon^{(a)}(x) d x .
$$

To compute the dispersion relation $\varepsilon^{(a)}\left(p^{(a)}\right)$ one has to eliminate the auxiliary variable $\lambda^{(a)}$ which connects energy and momentum. This is done by first computing the integrals in Eq. (16) with the help of the roots densities [Eq. (14)]. We then are able to eliminate the rapidity $\lambda^{(a)}$ from $\varepsilon^{(a)}\left(\lambda^{(a)}\right)$ and the final results for the dispersion relations are

$$
\begin{gathered}
\varepsilon^{(1)}\left(p^{(1)}\right)=4 \cos (\gamma) \sin \left(\frac{p^{(1)}}{2}\right) \sqrt{\sin ^{2}\left(\frac{p^{(1)}}{2}\right)+\tan ^{2}(\gamma)} \\
\varepsilon^{(2)}\left(p^{(2)}\right)=2 \sin (\gamma) \sin \left(p^{(2)}\right)
\end{gathered}
$$

Note that the dispersion relation associated to particle number excitations $\varepsilon^{(1)}\left(p^{(1)}\right)$ has the interesting feature of being factorized in terms of two physically distinct types of dispersions. The first part has a massless behavior while the second one has a massive character with a mass term proportional to $\tan (\gamma)$. We see that near the point $\gamma=0$ the mass gap becomes small and the terms depending on the momenta dominate Eq. (17). This means that at low energies the dispersion relation can crossover from a linear to a parabolic dependence on the momenta as $\gamma$ approaches zero. This is indeed the situation since we shall see below that the expression (17) remains valid down to $\gamma=0$. By way of contrast the dispersion related to the spin branch $\varepsilon^{(2)}\left(p^{(2)}\right)$ is similar to the spin-waves of the antiferromagnetic Heisenberg $X X Z$ model. For low momenta and as long as $\gamma \neq 0$ both charge and spin excitations behave linearly with the respective momenta. Their dispersion relations have in fact a common slope at $p^{(a)}=0$, namely,

$$
\varepsilon^{(a)}\left(p^{(a)}\right) \sim 2 \sin (\gamma) p^{(a)} \text { for } 0<\gamma \leq \frac{\pi}{2}
$$

and therefore they travel with the same speed of sound $v_{s}$ $=2 \sin \gamma$.

Let us turn our attention to the physical properties of the model at special point $\gamma=0$. In this case, the Hamiltonian (1)-(3) commutes also with the number of local electrons pairs ${ }^{18}$ and it is proportional to the graded permutator,

$$
H(\gamma=0)=\sum_{j=1}^{L} \sum_{a, b=1}^{4}(-1)^{p_{a} p_{b}} e_{a b}^{(j)} \otimes e_{b a}^{(j+1)}-L
$$

where $e_{a b}^{(j)}$ denotes $4 \times 4$ Weyl matrices acting on the $j$ th site and the Grassmann parities are given by $p_{1}=0, p_{2}=1, p_{3}$ $=1$, and $p_{4}=0$.

The diagonalization of the Hamiltonian (20) by the Bethe ansatz was discussed in the literature since long ago. ${ }^{3,5} \mathrm{We}$ remark that the respective Bethe equations do not follow immediately from Eqs. (5) and (6) when $\gamma \rightarrow 0$ due to the peculiar pattern of the Bethe roots $\left\{\mu_{j}\right\}$. We find, however, that certain properties of the model at $\gamma=0$ can be inferred without the need of using its Bethe ansatz solution. This is done by first investigating the pattern of the ground state degeneracies of Hamiltonian (20) by means of exact diagonalization up to $L=12$. This study has revealed that the ground state sits in many different sectors whose total number of particles is either $L$ or $L \pm 1$. This tells us the ground state for a given $L$ is $4 L$-fold degenerated and that its energy and low-lying excitations can be computed from the particular simple sectors $N_{+}=L, N_{-}=0$ or $N_{+}=0, N_{-}=L$. Because these are typical ferromagnetic states the calculations are rather direct. Denoting by $p$ the momentum of an excitation with spin $\sigma=-$ over the state $N_{+}=L, N_{-}=0$ one finds that the corresponding energy is

$$
E(p)=-2 L+4 \sin ^{2}\left(\frac{p}{2}\right)
$$

where for a finite $L$ the momenta $p=\frac{2 \pi}{L} K, K=0, \ldots, L-1$.

From Eq. (21) we conclude that the ground state per site is $e_{\infty}(\gamma=0)=-2$ and that for low momenta $p$ the excitation energy are proportional to $p^{2}$. Therefore, the system has a nonrelativistic behavior in accordance with previous works in the literature. ${ }^{18,19}$ Interesting enough, we observe that such results can also be derived from Eqs. (15) and (17) by taking the limit $\gamma \rightarrow 0$. To obtain the ground state energy from Eq. (15) we first perform the change of variable $\omega \rightarrow \omega / \gamma$ and afterward take the $\gamma \rightarrow 0$ limit. On the other hand, the dispersion relation $\varepsilon(p)=4 \sin ^{2}(p / 2)$ follows directly from Eq. (17) by substituting $\gamma=0$.

We have now the basic ingredients to investigate in next section the finite-size effects in the spectrum of the electronic models (1)-(3) for $0<\gamma \leq \pi / 2$.

\section{CRITICAL PROPERTIES}

The results of the previous section suggests to us that the generalized Hubbard model (1)-(3) in the regime $0<\gamma$ $\leq \pi / 2$ is conformally invariant. This means that the corresponding critical properties can be evaluated investigating the eigenspectrum finite-size corrections. ${ }^{21,22}$ For periodic boundary conditions, the ground state $E_{0}(L, \gamma)$ are expected to scale as

$$
\frac{E_{0}(L, \gamma)}{L}=e_{\infty}-\frac{\pi v_{s}(\gamma) c}{6 L^{2}}+O\left(L^{-2}\right)
$$

where $c$ is the central charge. 
From the excited states $E_{\alpha}(L, \gamma)$ we are able to determine the dimensions $X_{\alpha}(\gamma)$ of the respective primary operators, namely,

$$
\frac{E_{\alpha}(L, \gamma)}{L}-\frac{E_{0}(L, \gamma)}{L}=\frac{2 \pi v_{s}(\gamma) X_{\alpha}(\gamma)}{L^{2}}+O\left(L^{-2}\right) .
$$

A first insight on the structure of the finite-size corrections can be obtained by applying the so-called density root method. ${ }^{23-25}$ This approach explores the Bethe ansatz solution and it makes possible to compute the $O\left(L^{-2}\right)$ corrections to the densities of roots $\rho^{(a)}\left(\lambda^{(a)}\right)$. This method is however only suitable for systems whose ground state and low-lying excitations are described by real roots. Fortunately, this is exactly the situation we have found in Sec. II once the second root is shifted by $i \pi / 2$. Considering this subtlety on the root density approach we find that the leading finite-size behavior of the eigenenergies is

$$
\frac{E(L, \gamma)}{L}=e_{\infty}(\gamma)+\frac{2 \pi}{L^{2}} v_{s}(\gamma)\left[-\frac{1}{6}+X_{n_{+}, n_{-}}^{m, m_{-}}(\gamma)\right]+O\left(L^{-2}\right),
$$

where the dependence of the scaling dimensions $X_{n_{+}, n_{-}}^{m, m_{-}}(\gamma)$ on the anisotropy $\gamma$ is

$$
\begin{aligned}
X_{n_{+}, n_{-}}^{m, m_{-}}(\gamma)= & \frac{1}{4}\left[n_{+}^{2}+n_{-}^{2}+2\left(1-\frac{2 \gamma}{\pi}\right) n_{+} n_{-}\right]+\frac{\pi^{2}}{4 \gamma(\pi-\gamma)} \\
& \times\left[m^{2}+m_{-}^{2}-2\left(1-\frac{2 \gamma}{\pi}\right) m m_{-}\right] .
\end{aligned}
$$

As before the integers $n_{ \pm}$parameterizes the numbers of electrons $N_{+}=L / 2-n_{ \pm}$with spin $\sigma= \pm$. The indices $m=m_{+}$ $+m_{-}$and $m_{+}$characterize the presence of holes in the $Q_{j}^{(1)}$ and $Q_{j}^{(2)}$ distributions and in principle can be integers or half-integers. This approach is however not able to predict either the possible values for the vortex numbers $m$ and $m_{+}$ as well possible constraints with the corresponding spinwave integers $n$ and $n_{+}$. To shed some light on this problem we shall first study the finite-size effects at the particular point $\gamma=\pi / 2$. For $\gamma=\pi / 2$ we see that all the interactions in Hamiltonian (1)-(3) cancel out and we remain with two coupled free fermion models. In this case standard Fourier technique is able to provide us the exact expressions for the low-lying energies in the case of arbitrary $L$. Due to periodic boundary conditions, the respective calculations depend on the total number of electrons on the lattice $L$. We find that when $n=n_{+}+n_{-}$is odd that the expression for the lowest energy in this sector is given by

$$
E_{\text {odd }}\left(L, \frac{\pi}{2}\right)=-2 \frac{\left[\cos \left(\frac{\pi n_{+}}{L}\right)+\cos \left(\frac{\pi n_{-}}{L}\right)\right]}{\sin \left(\frac{\pi}{L}\right)} .
$$

Considering the asymptotic expansion of Eq. (26) for large $L$ one finds,

$$
\begin{aligned}
\frac{E_{\text {odd }}\left(L, \frac{\pi}{2}\right)}{L}= & e_{\infty}\left(\frac{\pi}{2}\right)+\frac{2 \pi}{L^{2}} v_{s}\left(\frac{\pi}{2}\right)\left[-\frac{1}{6}+\frac{n_{+}^{2}+n_{-}^{2}}{4}\right] \\
& +O\left(L^{-2}\right) .
\end{aligned}
$$

By way of contrast when $n=n_{+}+n_{-}$is an even number the lowest energy is

$$
E_{\text {even }}\left(L, \frac{\pi}{2}\right)=-\sum_{\sigma= \pm} \frac{\cos \left[\frac{\pi\left(n_{\sigma}+1\right)}{L}\right]}{\sin \left(\frac{\pi}{L}\right)}+\frac{\cos \left[\frac{\pi\left(n_{\sigma}-1\right)}{L}\right]}{\sin \left(\frac{\pi}{L}\right)},
$$

whose expansion for large $L$ is

$$
\begin{aligned}
\frac{E_{\text {even }}\left(L, \frac{\pi}{2}\right)}{L}= & e_{\infty}\left(\frac{\pi}{2}\right)+\frac{2 \pi}{L^{2}} v_{s}\left(\frac{\pi}{2}\right)\left[-\frac{1}{6}+\frac{n_{+}^{2}+n_{-}^{2}}{4}+\frac{1}{2}\right] \\
& +O\left(L^{-2}\right) .
\end{aligned}
$$

Taking into account Eqs. (27) and (29) we see that the expected finite-size corrections depend whether the index $n$ is an odd or even integer. In addition, by comparing Eqs. (27) and (29) with the general results (24) and (25) at $\gamma$ $=\pi / 2$ we clearly see that for $n$ odd the numbers $m$ and $m_{-}$ appear to start from zero while for $n$ even the lowest allowed value for $m$ and $m_{-}$is in fact one-half. This analysis strongly suggests that possible values for the vortex numbers $m$ and $m_{+}$should satisfy the following rule:

$$
\text { (1) for } n \text { odd } \rightarrow m, m_{-}=0, \pm 1, \pm 2, \ldots \text {, }
$$$$
\text { (2) for } n \text { even } \rightarrow m, m_{-}= \pm \frac{1}{2}, \pm \frac{3}{2}, \pm \frac{5}{2}, \ldots
$$

Let us now check if the above proposal remains valid for other values of the parameter $\gamma$. This is done mostly by solving numerically the original Bethe equations (5) and (6) up to $L=32$. For the excited states whose respective Bethe roots are unstable already for moderate values of $L$ we have used the data obtained from the numerical diagonalization through the Lanczos method. This numerical work enables us to compute for each $L$ the following sequence:

$$
X(L)=\left(\frac{E(L, \gamma)}{L}-e_{\infty}(\gamma)\right) \frac{L^{2}}{2 \pi v_{s}(\gamma)}+\frac{1}{6} .
$$

By extrapolating $X(L)$ for several values of $L$ we are able to verify expression (25) for $X_{n_{+}, n_{-}}^{m, m_{-}}(\gamma)$ and constraints (30). In Tables I-III we exhibit the finite-size sequence [Eq. (31)] for six lowest dimensions on the even sector to make an extensive check of the less unusual part of rule (30). For sake of completeness we also present three conformal dimensions corresponding to the $n$ odd sector. All those numerical results confirm conjectures (25) and (30) for the finite-size properties of the generalized Hubbard models (1)-(3).

We shall now proceed with a discussion of the results obtained so far. From Sec. II we know that the ground state 
TABLE I. Finite-size sequences [Eq. (31)] of the anomalous dimensions for $\gamma=\pi / 5, \pi / 3$ from the Bethe ansatz. The expected exact conformal dimensions are $X_{0,0}^{\frac{1}{2}, \frac{1}{2}}(\gamma)=\frac{1}{4(1-\gamma / \pi)}, X_{0,0}^{\frac{1}{2},-\frac{1}{2}}(\gamma)=\frac{1}{4(\gamma / \pi)}$, and $X_{1,-1}^{\frac{1}{2}, \frac{1}{2}}(\gamma)=\frac{\gamma}{\pi}$ $+\frac{1}{4(1-\gamma / \pi)}$.

\begin{tabular}{lcccccc}
\hline \hline$L$ & $X_{0,0}^{\frac{1}{2}, \frac{1}{2}\left(\frac{\pi}{5}\right)}$ & $X_{0,0}^{\frac{1}{2},-\frac{1}{2}\left(\frac{\pi}{5}\right)}$ & $X_{1,-1}^{\frac{1}{2}, \frac{1}{2}\left(\frac{\pi}{5}\right)}$ & $X_{0,0}^{\frac{1}{2}, \frac{1}{2}\left(\frac{\pi}{3}\right)}$ & $X_{0,0}^{\frac{1}{2},-\frac{1}{2}\left(\frac{\pi}{3}\right)}$ & $X_{1,-1}^{\frac{1}{2}, \frac{1}{2}}\left(\frac{\pi}{3}\right)$ \\
\hline 8 & 0.313380 & 1.227954 & 0.488672 & 0.380231 & 0.752958 & 0.681073 \\
12 & 0.312980 & 1.239120 & 0.498637 & 0.377310 & 0.751250 & 0.694030 \\
16 & 0.312787 & 1.243526 & 0.502997 & 0.376297 & 0.750687 & 0.699292 \\
20 & 0.312689 & 1.245526 & 0.505395 & 0.375829 & 0.750434 & 0.701996 \\
24 & 0.312633 & 1.247012 & 0.506892 & 0.375575 & 0.750299 & 0.703591 \\
28 & 0.312595 & 1.247954 & 0.507906 & 0.375423 & 0.750218 & 0.704619 \\
32 & 0.312576 & 1.248063 & 0.508633 & 0.375323 & 0.750166 & 0.705327 \\
Extrap. & $0.31249 \pm 1$ & $1.2504 \pm 2$ & $0.51219 \pm 1$ & $0.37498 \pm 1$ & $0.74999 \pm 1$ & $0.708336 \pm 1$ \\
Exact & 0.3125 & 1.25 & 0.5125 & 0.375 & 0.75 & $0.70833 \cdots$ \\
\hline \hline
\end{tabular}

sits in the sectors $n_{+}= \pm 1$ and $n_{-}=0$ or $n_{+}=0$ and $n_{-}= \pm 1$. Considering rule (30) the corresponding vortex numbers have the lowest possible values $m=m_{+}=0$ and from Eqs. (24) and (25) we derive the following finite-size behavior:

$$
\frac{E_{0}(L, \gamma)}{L}=e_{\infty}+\frac{\pi v_{s}(\gamma)}{6 L^{2}}+O\left(L^{-2}\right)
$$

Direct comparison between Eqs. (22) and (32) leads us to conclude that the central charge of the underlying conformal theory is

$$
c=-1 \text { for } 0<\gamma \leq \frac{\pi}{2} \text {. }
$$

Recall here that central charge [Eq. (33)] has been derived from the finite-size corrections with strict periodic boundary conditions. In the operator content of conformal theories with $c \leq 0$ it is however expected the presence of negative conformal weights. This means that if $X_{<}$is the lowest such scaling dimension the true ground state is then governed by the effective central $c_{e f}=c-12 X_{<}$. This type of sector in models with underlying superalgebra can be obtained by considering antiperiodic boundary conditions for the fermi- onic degrees of freedom, see for instance. ${ }^{26}$ The antiperiodic twist prompts the sector with maximum number of Bethe roots $n_{ \pm}=0$ which is now compatible with the vortex quantum numbers $m=m_{-}=0$. Denoting by $E_{<}(L, \gamma)$ the energy corresponding to this state we derive from the Eqs. (24) and (25) the following scaling behavior:

$$
\frac{E_{<}(L, \gamma)}{L}-\frac{E_{0}(L, \gamma)}{L}=\frac{2 \pi v_{s}(\gamma)}{L^{2}}\left(-\frac{1}{4}\right)+O\left(L^{-2}\right) .
$$

From Eq. (34) we see that the negative scaling dimension is $X_{<}=-\frac{1}{4}$ and the respective effective central charge is therefore $c_{e f}=-1+\frac{12}{4}=2$. It is expected that the behavior of the heat capacity at low-temperature $T$ be governed by such effective central charge. ${ }^{21}$ This means that the leading term of the heat capacity per length of such electronic model should be $\frac{2 \pi T}{3 v_{s}(\gamma)}$ for $0<\gamma \leq \pi / 2$.

Let us now conclude by discussing the conformal dimensions on the periodic sector. The scaling dimensions of the primary operators $\bar{X}_{n, n_{+}}^{m, m_{+}}(\gamma)$ depend on the anisotropy $\gamma$ and they should be measured from the ground state $E_{0}(L, \gamma)$. Considering Eqs. (24) and (25) together with Eq. (32) we find that they are given by

TABLE II. Finite-size sequences [Eq. (31)] of the anomalous dimensions for $\gamma=\pi / 5, \pi / 3$ from the Bethe ansatz. The exact conformal dimensions are $X_{1,0}^{0,0}(\gamma)=\frac{1}{4}, X_{2,-1}^{0,0}(\gamma)=\frac{1}{4}+\frac{2 \gamma}{\pi}$, and $X_{2,-2}^{\frac{1}{2}, \frac{1}{2}}(\gamma)=\frac{4 \gamma}{\pi}+\frac{1}{4(1-\gamma / \pi)}$.

\begin{tabular}{lcccccc}
\hline \hline$L$ & $X_{1,0}^{0,0}\left(\frac{\pi}{5}\right)$ & $X_{2,-1}^{0,0}\left(\frac{\pi}{5}\right)$ & $X_{2,-2}^{\frac{1}{2}, \frac{1}{2}}\left(\frac{\pi}{5}\right)$ & $X_{1,0}^{0,0}\left(\frac{\pi}{3}\right)$ & $X_{2,-1}^{0,0}\left(\frac{\pi}{3}\right)$ & $X_{2,-2}^{\frac{1}{2}, \frac{1}{2}}\left(\frac{\pi}{3}\right)$ \\
\hline 8 & 0.251098 & 0.642630 & 1.000395 & 0.252587 & 0.902529 & 1.541194 \\
12 & 0.250523 & 0.646574 & 1.047807 & 0.251149 & 0.910140 & 1.622978 \\
16 & 0.250301 & 0.648003 & 1.068757 & 0.250646 & 0.912924 & 1.655748 \\
20 & 0.250195 & 0.648689 & 1.080196 & 0.250413 & 0.914244 & 1.672266 \\
24 & 0.250136 & 0.649072 & 1.087265 & 0.250287 & 0.914971 & 1.681827 \\
28 & 0.250101 & 0.649307 & 1.092001 & 0.250211 & 0.915414 & 1.687896 \\
32 & 0.250077 & 0.649463 & 1.095375 & 0.250161 & 0.915704 & 1.692009 \\
Extrap. & $0.250003 \pm 1$ & $0.65003 \pm 1$ & $1.1124 \pm 1$ & $0.250004 \pm 2$ & $0.9167 \pm 2$ & $1.70825 \pm 1$ \\
Exact & 0.25 & 0.65 & 1.1125 & 0.25 & $0.91666 \cdots$ & $1.70833 \cdots$ \\
\hline
\end{tabular}


TABLE III. Finite-size sequences [Eq. (31)] of the anomalous dimensions for $\gamma=\pi / 5, \pi / 4$ from Lanczos. The expected exact conformal dimensions are $X_{1,0}^{1,0}(\gamma)=\frac{1}{4}+\frac{1}{4(\gamma / \pi)(1-\gamma / \pi)}, X_{1,1}^{\frac{1}{2}}, \frac{1}{2}(\gamma)=(1-\gamma / \pi)+\frac{1}{4(1-\gamma / \pi)}$, and $X_{1,1}^{\frac{1}{2},-\frac{1}{2}}(\gamma)=(1-\gamma / \pi)+\frac{1}{4(\gamma / \pi)}$.

\begin{tabular}{lcccccc}
\hline$L$ & $X_{1,0}^{1,0}\left(\frac{\pi}{5}\right)$ & $X_{1,1}^{\frac{1}{2}, \frac{1}{2}\left(\frac{\pi}{5}\right)}$ & $X_{1,1}^{\frac{1}{2},-\frac{1}{2}\left(\frac{\pi}{5}\right)}$ & $X_{1,0}^{1,0}\left(\frac{\pi}{4}\right)$ & $X_{1,1}^{\frac{1}{2}, \frac{1}{2}}\left(\frac{\pi}{4}\right)$ & $X_{1,1}^{\frac{1}{2},-\frac{1}{2}}\left(\frac{\pi}{4}\right)$ \\
\hline 4 & 1.497964 & 1.395242 & 1.426027 & 1.355168 & 1.225412 & 1.261369 \\
6 & 1.661634 & 1.288542 & 1.741570 & 1.480050 & 1.172763 & 1.563850 \\
8 & 1.724314 & 1.224764 & 1.888707 & 1.523739 & 1.140033 & 1.639713 \\
10 & 1.754379 & 1.188829 & 1.947347 & 1.544502 & 1.121710 & 1.679436 \\
12 & 1.771238 & 1.167328 & 1.977593 & 1.556018 & 1.110818 & 1.699900 \\
14 & 1.781660 & 1.153629 & 1.995956 & 1.563070 & 1.103911 & 1.712660 \\
16 & 1.788561 & 1.144426 & 2.008012 & 1.567703 & 1.098013 & 1.721046 \\
Extrap. & $1.812 \pm 1$ & $1.12 \pm 1$ & $2.06 \pm 1$ & $1.583 \pm 1$ & $1.079 \pm 1$ & $1.73 \pm 1$ \\
Exact & 1.8125 & 1.1125 & 2.05 & $1.5833 \cdots$ & $1.0833 \cdots$ & 1.75 \\
\hline \hline
\end{tabular}

$$
\bar{X}_{n, n_{+}}^{m, m_{+}}(\gamma)=X_{n, n_{+}}^{m, m_{+}}(\gamma)-\frac{1}{4} \quad \text { for } 0<\gamma \leq \frac{\pi}{2} .
$$

To our knowledge, models exhibiting this kind of universality class have so far been found in a not self-adjoint theory based on the deformed $\operatorname{osp}(2 \mid 2)$ symmetry. ${ }^{27}$ Therefore, the correlated electron system (1)-(3) appears to be the first example of a Hermitian Hamiltonian whose continuum limit is described by a field theory with $c=-1$ with continuously varying anomalous dimensions. The fact that a line of critical exponents with $c<0$ can be realized in terms of Hermitian models could be of importance for practical applications in condensed matter such as in the physics of disordered systems.

\section{CONCLUSIONS}

We have studied the thermodynamic limit and the finitesize behavior of an exactly solvable generalization of the Hubbard model with free parameter $\gamma$ related to the quantum $U_{q}[\operatorname{SU}(2 \mid 2)$ superalgebra where $q=\exp (i \gamma)$. We have determined the nature of the ground state and the behavior of the elementary excitations. One interesting feature of the model is that as $\gamma$ approaches zero the spectrum associated with several distinct sectors degenerate to that of the ferromagnetic sector $N_{ \pm}=L$. Interesting enough, this behavior is somehow encoded on the structure of the dispersion relation for the charge sector. It has the peculiar feature of being given in terms of the product of massless and massive energy-momenta relations. When $\gamma \rightarrow 0$ the mass gap goes to zero and the dependence on the momenta prevail in the dis- persion relation. As a result, we clearly observe that charge dispersion relation changes its dependence on low momenta from linear to parabolic. The latter being a characteristic of ferromagnetic behavior.

In the regime $0<\gamma \leq \pi / 2$ the low-lying excitations have relativistic behavior and they travel with the same speed of sound $v_{s}(\gamma)=2 \sin (\gamma)$. The underlying conformal theory has central charge $c=-1$ and a line of continuously varying exponents. We stress that conformal theories with $c \leq 0$ are expected to have negative conformal weights and the true ground state is then governed by the effective central charge. In our case we found that such effective central charge is $c_{e f}=2$ predicting that the leading behavior of the heat capacity should be $\frac{2 \pi T}{3 v_{s}(\gamma)}$ for $0<\gamma \leq \pi / 2$.

For the particular point $\gamma=0$ we have argued that basic properties can be obtained without recourse to the Bethe ansatz solution. We expect that this observation remains valid for all integrable models based on the Lie superalgebra $\operatorname{sl}(p \mid q)$ for arbitrary finite number of bosonic $(p)$ and fermionic $(q)$ degrees of freedom. This suggests that the models based on the deformed $\operatorname{sl}(p \mid q)$ symmetry may also have excitation modes with dispersion relation exhibiting both massless and massive behaviors. We hope to investigate this interesting possibility as well as its consequences in a future publication.

\section{ACKNOWLEDGMENTS}

This work was supported by the Brazilian Research Agencies $\mathrm{CNPq}$ and FAPESP.
${ }^{1}$ F. D. M. Haldane, Phys. Rev. Lett. 45, 1358 (1980); J. Phys. C 14, 2585 (1981).

${ }^{2}$ H. E. Lieb and F. Y. Wu, Phys. Rev. Lett. 20, 1445 (1968).

${ }^{3}$ B. Sutherland, Phys. Rev. B 12, 3795 (1975).

${ }^{4}$ P. Schlottmann, Phys. Rev. B 36, 5177 (1987); Int. J. Mod. Phys.
B 11, 355 (1997).

${ }^{5}$ P. P. Kulish, J. Sov. Math. 35, 2648 (1986).

${ }^{6}$ F. H. L. Essler, V. E. Korepin, and K. Schoutens, Phys. Rev. Lett. 68, 2960 (1992); 70, 73 (1993).

${ }^{7}$ A. J. Bracken, M. D. Gould, J. R. Links, and Y.-Z. Zhang, Phys. 
Rev. Lett. 74, 2768 (1995).

${ }^{8}$ P. Fendley, K. Schoutens, and J. de Boer, Phys. Rev. Lett. 90, 120402 (2003); P. Fendley, B. Nienhuis, and K. Schoutens, J. Phys. A 36, 12399 (2003).

${ }^{9}$ P. Fendley and K. Schoutens, Phys. Rev. Lett. 95, 046403 (2005); M. Beccaria and G. F. De Angelis, ibid. 94, 100401 (2005); H. van Eerten, J. Math. Phys. 46, 123302 (2005).

${ }^{10}$ L. Huijse and K. Schoutens, Eur. Phys. J. B 64, 543 (2008); arXiv:0910.2386 (unpublished).

${ }^{11}$ I. N. Karnaukhov, Phys. Rev. Lett. 73, 1130 (1994); Phys. Rev. B 51, 7858 (1995).

${ }^{12}$ G. Bedurftig and H. Frahm, J. Phys. A 28, 4453 (1995).

${ }^{13}$ R. Z. Bariev, A. Klümper, and J. Zittartz, Europhys. Lett. 32, 85 (1995); M. D. Gould, K. E. Hibberd, J. R. Links, and Y.-Z. Zhang, Phys. Lett. A 212, 156 (1996).

${ }^{14}$ M. D. Gould, J. R. Links, Y.-Z. Zhang, and I. Tsonhantjis, J. Phys. A 30, 4313 (1997).

${ }^{15}$ M. J. Martins and P. B. Ramos, Phys. Rev. B 56, 6376 (1997).

${ }^{16}$ H. Saleur,J. Phys. A 32, L207 (1999).

${ }^{17}$ N. Beisert and P. Koroteev, J. Phys. A: Math. Theor. 41, 255204 (2008); F. C. Alcaraz and R. Z. Bariev, J. Phys. A 32, L483 (1999).
${ }^{18}$ F. H. L. Eßler and V. E. Korepin, Int. J. Mod. Phys. B 8, 3243 (1994).

${ }^{19}$ H. Saleur, Nucl. Phys. B 578, 552 (2000).

${ }^{20}$ L. D. Faddeev and L. A. Takhtajan, Phys. Lett. A 85, 375 (1981); J. Sov. Math. 24, 241 (1984).

${ }^{21}$ H. W. J. Blote, J. L. Cardy, and M. P. Nightingale, Phys. Rev. Lett. 56, 742 (1986); I. Affleck, ibid. 56, 746 (1986).

${ }^{22}$ J. L. Cardy, in Phase Transitions and Critical Phenomena, edited by C. Domb and L. J. Lebowitz (Academic Press, New York, 1987), Vol. 11.

${ }^{23}$ H. J. de Vega and F. Woynarovich, Nucl. Phys. B 251, 434 (1985); F. Woynarovich and H.-P. Eckle, J. Phys. A 20, L443 (1987); N. M. Bogoliubov, A. G. Izergin, and N. Yu. Reshetikhin, ibid. 20, 5361 (1987).

${ }^{24}$ H. J. de Vega, J. Phys. A 21, L1089 (1988); J. Suzuki, ibid. 21, L1175 (1988).

${ }^{25}$ H. J. de Vega and E. Lopes, Phys. Rev. Lett. 67, 489 (1991).

${ }^{26}$ M. J. Martins, B. Nienhuis, and R. Rietman, Phys. Rev. Lett. 81, 504 (1998); J. L. Jacobsen, N. Read, and H. Saleur, ibid. 90, 090601 (2003); F. H. L. Essler, H. Frahm, and H. Saleur, Nucl. Phys. B 712, 513 (2005).

${ }^{27}$ W. Galleas and M. J. Martins, Nucl. Phys. B 768, 219 (2007). 